Notre Dame Journal of Formal Logic

Volume 47, Number 3, 2006

\title{
Andersonian Deontic Logic, Propositional Quantification, and Mally
}

\author{
Gert-Jan C. Lokhorst
}

\begin{abstract}
We present a new axiomatization of the deontic fragment of Anderson's relevant deontic logic, give an Andersonian reduction of a relevant version of Mally's deontic logic previously discussed in this journal, study the effect of adding propositional quantification to Anderson's system, and discuss the meaning of Anderson's propositional constant in a wide range of Andersonian deontic systems.
\end{abstract}

\section{Introduction}

An Andersonian system of deontic logic is a system in which the deontic operator $O$ ("it is obligatory that") is defined by $O A=e \Rightarrow A$, where $e$ is a primitive propositional constant ("the good thing") and $\Rightarrow$ is an implicational connective. The following systems are examples of Andersonian deontic systems.

1. The systems discussed in [1] and [2], in which $\Rightarrow$ is strict implication, that is, $O A=\square(e \rightarrow A)$, where $\square$ is the modal operator of necessity and $\rightarrow$ is material implication.

2. Anderson's relevant deontic logic ([3], [4]), in which $\Rightarrow$ is relevant implication.

3. The systems discussed in [8] and [14], in which $\Rightarrow$ is strict relevant implication, that is, $O A=\square(e \rightarrow A)$, where $\square$ is necessity and $\rightarrow$ is relevant implication.

In this paper, we will present some new results on Andersonian deontic systems. We start with Anderson's relevant deontic logic. We give a new axiomatization of the deontic fragment of this system, show that the relevant version of Mally's deontic logic [13] presented in [11] is an extension of this fragment, and prove that $e$ can be defined in terms of $O$ as soon as propositional quantification is available. After

Received September 2, 2005; accepted January 2, 2006; printed November 14, 2006 2000 Mathematics Subject Classification: Primary, 03B47; Secondary, 03B45

Keywords: Anderson, deontic logic, relevance logic, propositional quantification, Mally (c)2006 University of Notre Dame 
this, we discuss some of the other Andersonian systems mentioned above. We will show that the addition of propositional quantification sheds light on the meaning of the constant $e$ in these systems, too.

\section{Anderson's Relevant Deontic Logic}

Definition 2.1 (System R) Relevant system $\mathbf{R}$ has the following axioms and rules ([5], Ch. V).

$\begin{array}{lll}\text { (R1) } & A \rightarrow A & \text { (Self-implication) } \\ \text { (R2) } & (A \rightarrow B) \rightarrow((C \rightarrow A) \rightarrow(C \rightarrow B)) & \text { (Prefixing) } \\ \text { (R3) } & (A \rightarrow(B \rightarrow C)) \rightarrow(B \rightarrow(A \rightarrow C)) & \text { (Permutation) } \\ \text { (R4) } & (A \rightarrow(A \rightarrow B)) \rightarrow(A \rightarrow B) & \text { (Contraction) } \\ \text { (R5) } & (A \& B) \rightarrow A,(A \& B) \rightarrow B & \text { (\&Elimination) } \\ \text { (R6) } & ((A \rightarrow B) \&(A \rightarrow C)) \rightarrow(A \rightarrow(B \& C)) & \text { (\&Introduction) } \\ \text { (R7) } & A \rightarrow(A \vee B), B \rightarrow(A \vee B) & \text { (VIntroduction) } \\ \text { (R8) } & ((A \rightarrow C) \&(B \rightarrow C)) \rightarrow((A \vee B) \rightarrow C) & \text { (VElimination) } \\ \text { (R9) } & (A \&(B \vee C)) \rightarrow((A \& B) \vee C) & \text { (Distribution) } \\ \text { (R10) } & \neg \neg A \rightarrow A & \text { (Double Negation) } \\ \text { (R11) } & (A \rightarrow \neg B) \rightarrow(B \rightarrow \neg A) & \text { (Contraposition) } \\ (\rightarrow \mathrm{E}) & \text { If } A \text { and } A \rightarrow B \text { are theorems, } B \text { is a theorem } & \text { (Detachment) } \\ \text { (\&I) } & \text { If } A \text { and } B \text { are theorems, } A \& B \text { is a theorem } & \text { (Adjunction) }\end{array}$

Definition: $A \leftrightarrow B=(A \rightarrow B) \&(B \rightarrow A)$.

Definition 2.2 (System $\mathbf{R}_{e}$ ) Anderson's relevant deontic logic $\mathbf{R}_{e}$ is $\mathbf{R}$ with a primitive propositional constant $e$ and a unary operator $O$ defined by $O A=e \rightarrow A$. Furthermore, the operator $P$ is defined by $P A=\neg O \neg A$. $e$ is read as "the good thing," $O$ as "it is obligatory that," and $P$ as "it is permitted that."

\section{The Deontic Fragment of Anderson's Relevant Deontic Logic}

To which "purely deontic" logic, stated in terms of $O$ rather than $e$, does Anderson's proposal exactly give rise? This question has been answered by Goble [8].

Definition 3.1 (System OR.1abc) Language: $\mathbf{R}$ supplemented with a primitive propositional operator $O$. Axioms and rules: R plus:

$(\mathrm{OC}) \quad(O A \& O B) \rightarrow O(A \& B)$

(OK) $\quad O(A \rightarrow B) \rightarrow(O A \rightarrow O B)$

(ROa) If $A \rightarrow B$ is a theorem, then so is $O A \rightarrow O B$

(a) $O(O A \rightarrow A)$

(b) $(A \rightarrow B) \rightarrow(O A \rightarrow O B)$

(c) $\quad A \rightarrow O P A$

Definition 3.2 (Deontic Fragment) The translation function $h$ from the language of OR.1abc into the language of $\mathbf{R}_{e}$ is defined as follows.

1. $h(A)=A$ if $A$ is atomic

2. $h(\neg A)=\neg h(A)$

3. $h(A \& B)=h(A) \& h(B)$

4. $h(A \vee B)=h(A) \vee h(B)$

5. $h(A \rightarrow B)=h(A) \rightarrow h(B)$

6. $h(O A)=e \rightarrow h(A)$ 
The deontic fragment of $\mathbf{R}_{e}$ (under $h$ ) is the set $\left\{A: \vdash \mathbf{R}_{e} h(A)\right\}$.

Theorem 3.3 OR.1abc is an axiomatization of the deontic fragment of $\mathbf{R}_{e}$.

Proof By the Routley-Meyer semantics of $\mathbf{R}_{e}$ and OR.1abc. See [8] for details. ${ }^{2}$

This result can be simplified, as we will now show.

Definition 3.4 (System $\mathbf{R}_{O}$ ) Language: $\mathbf{R}$ supplemented with a primitive propositional operator $O$. Axioms and rules: $\mathbf{R}$ plus:

(a) $O(O A \rightarrow A)$

(b) $(A \rightarrow B) \rightarrow(O A \rightarrow O B)$

Theorem 3.5 $\quad \mathbf{R}_{O}$ has the same theorems as OR.1abc.

Proof It is sufficient to prove that (ROa) is a derivable rule of $\mathbf{R}_{O}$ and that (c), (OK), and (OC) are theorems of $\mathbf{R}_{O}$.

The proofs are as follows. We mention a few intermediate theorems for later reference. To avoid circularity, there are no forward references in this section.

(ROa) From (b) and detachment.

(Th1) $O(A \rightarrow B) \rightarrow(A \rightarrow O B)$.

$$
\begin{array}{lll}
\text { 1. } & (A \rightarrow B) \rightarrow(A \rightarrow B) & \text { self-impl } \\
\text { 2. } & A \rightarrow(A \rightarrow B) \rightarrow B) & 1 \text {, permut } \\
\text { 3. } & A \rightarrow(O(A \rightarrow B) \rightarrow O B) & 2 \text {, (b) } \\
\text { 4. } & O(A \rightarrow B) \rightarrow(A \rightarrow O B) & 3 \text {, permut }
\end{array}
$$

(c) $A \rightarrow O P A$.
1. $O(O \neg A \rightarrow \neg A)$
2. $(O \neg A \rightarrow \neg A) \rightarrow(A \rightarrow P A)$
3. $O(A \rightarrow P A)$
(a)
4. $A \rightarrow O P A$
contrapos, def $P$
$1,2,(b)$
3, (Th1)

(Th2) $(A \rightarrow O B) \rightarrow O(A \rightarrow B)$.
1. $\quad(O B \rightarrow B) \rightarrow((A \rightarrow O B) \rightarrow(A \rightarrow B))$
prefixing
2. $O(O B \rightarrow B) \rightarrow O((A \rightarrow O B) \rightarrow(A \rightarrow B))$
1 , (b)
3. $O((A \rightarrow O B) \rightarrow(A \rightarrow B))$
2 , (a)
4. $(A \rightarrow O B) \rightarrow O(A \rightarrow B)$
3, (Th1)

(ROO) If $O O A$ is a theorem, then $O A$ is a theorem. Definition (for readability): $D=(O A \rightarrow A)$.
1. $O O A$
2. $D \rightarrow(O O A \rightarrow O A)$
3. $D \rightarrow O A$
premise
4. $\quad D \rightarrow((D \rightarrow O A) \rightarrow(D \rightarrow A))$
def $D$, (b)
5. $\quad D \rightarrow(D \rightarrow A)$
1,2 , permut
def $D$, pref
6. $D \rightarrow A$
7. $O D \rightarrow O A$
3,4 , permut
5 , contract
8. $O D$
6, (b)
def $D$, (a)
9. $O A$
7, 8 


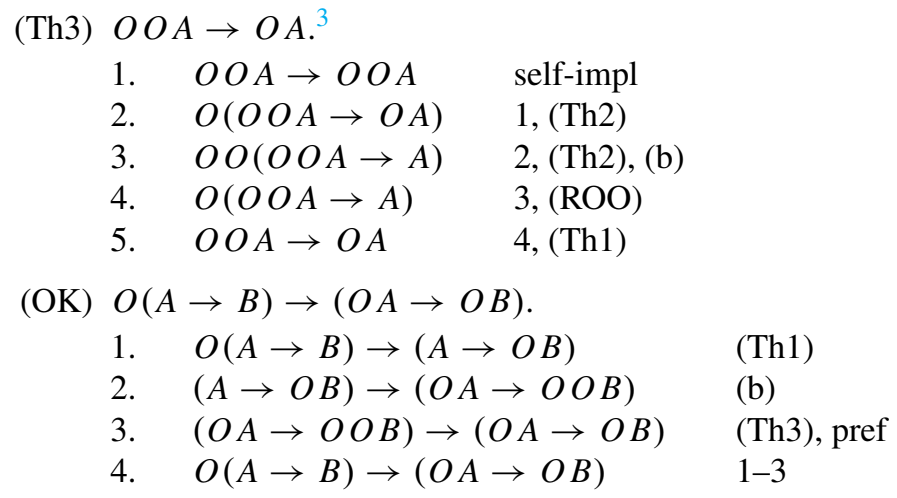

(Th4) $(A \rightarrow B) \rightarrow(P A \rightarrow P B)$. From axiom (b) and (R10)-(R11).

(Th5) $P O A \rightarrow A$. From theorem (c), axiom (b), and (R10)-(R11).

(OC) $(O A \& O B) \rightarrow O(A \& B) .^{4}$

$\begin{array}{lll}\text { 1. } & P(O A \& O B) \rightarrow P O A & \text { \&Elim, (Th4) } \\ \text { 2. } & P(O A \& O B) \rightarrow A & 1,(\mathrm{Th} 5) \\ \text { 3. } & P(O A \& O B) \rightarrow P O B & \text { \&Elim, (Th4) } \\ \text { 4. } & P(O A \& O B) \rightarrow B & 3,(\mathrm{Th} 5) \\ \text { 5. } & P(O A \& O B) \rightarrow(A \& B) & 2,4, \text { Introd } \\ \text { 6. } & O P(O A \& O B) \rightarrow O(A \& B) & 5,(\mathrm{~b}) \\ \text { 7. } & (O A \& O B) \rightarrow O(A \& B) & 6,(\mathrm{c})\end{array}$

This completes the proof.

Corollary 3.6 $\quad \mathbf{R}_{O}$ is an axiomatization of the deontic fragment of $\mathbf{R}_{e}$.

Interestingly enough, (OC) is not a theorem of positive $\mathbf{R}_{O}$, that is, positive $\mathbf{R}$ with axioms (a) and (b) (proof: by MaGIC [18]). ${ }^{5}$ In other words, $\mathbf{R}_{O}$ is not a conservative extension of positive $\mathbf{R}_{O}$. But we can state the following (by inspection of the proof in [8]).

Theorem 3.7 Positive $\mathbf{R}_{O}$ plus (OC) is an axiomatization of the deontic fragment of positive $\mathbf{R}_{e}$.

In contrast with Goble's system OR.1abc, systems $\mathbf{R}_{O}$ and positive $\mathbf{R}_{O}$ are well axiomatized in the sense that the axioms are independent from each other (proof: by MaGIC [18]).

\section{Mally's Deontic Logic}

In an earlier paper [11], we presented the following relevant version of Ernst Mally's deontic logic, the first formal system of deontic logic ever put forward [13].

Definition 4.1 (System RD (Relevant Deontik)) $\quad$ Language: relevant system R supplemented with a primitive unary operator $O$ and a primitive propositional constant $u$ ("the unconditionally obligatory"). Axioms and rules: $\mathbf{R}$ plus:

(I) $\quad((A \rightarrow O B) \&(B \rightarrow C)) \rightarrow(A \rightarrow O C)$

(II) $\quad((A \rightarrow O B) \&(A \rightarrow O C)) \rightarrow(A \rightarrow O(B \& C))$

(III) $\quad(A \rightarrow O B) \leftrightarrow O(A \rightarrow B)$

(IV) $O u$

(V) $\quad \neg(u \rightarrow O \neg u)$ 
This system is the same as Mally's own system, except that Mally based his system on classical logic and accordingly accepted the "archetypical fallacy of relevance" $A \rightarrow(B \rightarrow A)$. Mally's system has the theorem $A \leftrightarrow O A$ (see [17]), but neither $A \rightarrow O A$ nor $O A \rightarrow A$ are theorems of RD (proof: by MaGIC [18]).

It can be shown that RD can be axiomatized more elegantly as follows (see [12] in combination with the derivation of theorem (OC) above).

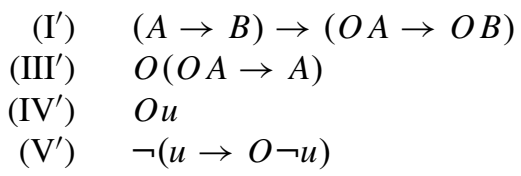

From the results we have presented above, it follows that RD is the deontic fragment of $\mathbf{R}_{e}$ with an additional propositional constant $u$ plus the axioms $e \rightarrow u$ and $\neg(u \rightarrow(e \rightarrow \neg u))$.

This result is useful because it makes it considerably easier to recognize some theorems of RD as such. For example, in [11] we failed to see that $O O A \rightarrow O A$ is a theorem, whereas we can now easily identify it as a theorem because $h(O O A \rightarrow O A)$, that is, $(e \rightarrow(e \rightarrow A)) \rightarrow(e \rightarrow A)$, is just an instance of Contraction.

\section{Propositional Quantification (1)}

What does the propositional constant $e$ exactly mean? The following interpretations have been offered: "the good thing" or "good state of affairs" ([1], [2], [3], [4]), "what morality requires" [10], "optimality or admissibility" [7], "the content of an (unspecified) moral code" [19], "the law," "it is not the case that all hell breaks loose" [20], "all normative demands are met" [16]. Most of these interpretations sound like poetry rather than logic. We shall show that once propositional quantifiers are added to $\mathbf{R}_{e}$, we can be more precise about the meaning of $e$.

Definition 5.1 (System $\mathbf{R}^{\forall p}$ ) Propositionally quantified relevant system $\mathbf{R}^{\forall p}$ has the following axioms and axiom clause in addition to those of $\mathbf{R}$ ([6], Ch. VI).

(Q1) $\quad \forall p(A \rightarrow B) \rightarrow(\forall p A \rightarrow \forall p B)$

(Q2) $\quad(\forall p A \& \forall p B) \rightarrow \forall p(A \& B)$

(Q3) $\quad \forall p A(p) \rightarrow A(B)$

(Q4) $\forall p(A \rightarrow B) \rightarrow(A \rightarrow \forall p B)(p$ not free in $A)$

(Q5) $\quad \forall p(A \vee B) \rightarrow(A \vee \forall p B)(p$ not free in $A)$

(Q*) If $A$ is an axiom, then $\forall p A$ is an axiom.

Axioms Q1 and Q2 in conjunction with axiom clause Q* yield Generalization:

(Gen) If $A$ is a theorem, then $\forall p A$ is a theorem.

Definition 5.2 (System $\mathbf{R}_{e}^{\forall p}$ ) $\quad \mathbf{R}_{e}^{\forall p}$ is $\mathbf{R}^{\forall p}$ with primitive propositional constant $e$ and propositional operators $O$ and $P$ defined as in $\mathbf{R}_{e}$.

Theorem 5.3 $\quad \mathbf{R}_{e}^{\forall p}$ has the following theorem: $e \leftrightarrow \forall p(O p \rightarrow p)$. 


\section{Proof}

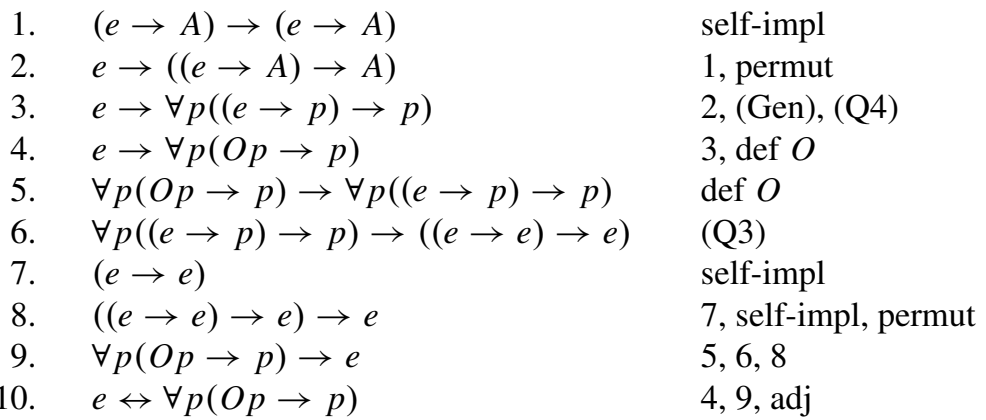

Thus $e$ says that all obligations are fulfilled (all normative demands are met). This happens to agree with McNamara's unmotivated informal reading of $e$ [16].

\section{Inverse Andersonian Reduction (1)}

Theorem 5.3 suggests the following question: is it possible to define $e$ in terms of $O$ and carry out the inverse of the Andersonian reduction? We shall show that this is indeed possible.

Definition 6.1 (System $\mathbf{R}_{O}^{\forall p}$ ) System $\mathbf{R}_{O}^{\forall p}$ is $\mathbf{R}^{\forall p}$ supplemented with a primive operator $O$, a propositional constant $e$ defined by $e=\forall p(O p \rightarrow p)$, and axioms (a) and (b) of $\mathbf{R}_{O}$.

Theorem 6.2 $\quad \mathbf{R}_{O}^{\forall p}$ and $\mathbf{R}_{e}^{\forall p}$ have the same theorems.

Proof First, all theorems of $\mathbf{R}_{O}^{\forall p}$ are theorems of $\mathbf{R}_{e}^{\forall p}$. All cases are easy except perhaps $e \leftrightarrow \forall p(O p \rightarrow p)$ (which is a theorem of $\mathbf{R}_{O}^{\forall p}$ because of the definition of $e$ in $\mathbf{R}_{O}^{\forall p}$ ), but this case has already been discussed (Theorem 5.3).

Second, all theorems of $\mathbf{R}_{e}^{\forall p}$ are theorems of $\mathbf{R}_{O}^{\forall p}$. It is sufficient to prove that $O A \leftrightarrow(e \rightarrow A)$ is a theorem of $\mathbf{R}_{O}^{\forall p}$. (This formula is a theorem of $\mathbf{R}_{e}^{\forall p}$ because of the definition of $O$ in $\mathbf{R}_{e}^{\forall p}$.)

Let us first derive the deontic Barcan formula:

$$
\forall p O A(p) \rightarrow O \forall p A(p)
$$

The following derivation is similar to the proof of (OC) above. It is also similar to a well-known proof of the Barcan formula in individually quantified S5 ([9], p. 247).
1. $\quad P \forall p O A(p) \rightarrow P O A(B)$
Q3, (Th4)
2. $\quad P \forall p O A(p) \rightarrow A(B)$
$1,(\mathrm{Th} 5)$
3. $\forall q(P \forall p O A(p) \rightarrow A(q))$
$2,(\mathrm{Gen})$
4. $\quad P \forall p O A(p) \rightarrow \forall p A(p)$
$3,(\mathrm{Q} 4)$
5. $O P \forall p O A(p) \rightarrow O \forall p A(p)$
4 , (b)
6. $\forall p O A(p) \rightarrow O \forall p A(p)$
$5,(\mathrm{c})$

We can now derive $O A \leftrightarrow(e \rightarrow A)$. 


$\begin{array}{lll}\text { 1. } & e \rightarrow(O A \rightarrow A) & \operatorname{def} e,(\mathrm{Q} 3) \\ \text { 2. } & O A \rightarrow(e \rightarrow A) & 1, \text { permut } \\ \text { 3. } & O(e \rightarrow A) \rightarrow(O e \rightarrow O A) & \text { (OK) } \\ \text { 4. } & O e \rightarrow((e \rightarrow A) \rightarrow O A) & 3, \text { permut } \\ \text { 5. } & \forall p O(O p \rightarrow p) & (\text { a), (Q*) } \\ \text { 6. } & O \forall p(O p \rightarrow p) & 5,(\mathrm{OBF}) \\ \text { 7. } & O e & 6, \operatorname{def} e \\ \text { 8. } & (e \rightarrow A) \rightarrow O A & 4,7 \\ \text { 9. } & O A \leftrightarrow(e \rightarrow A) & 2,8, \text { adj }\end{array}$

We might call this "a reduction of alethic logic to deontic logic."

\section{Weaker Andersonian Systems}

Some authors ([8], [15], [14]) have objected to axiom (b) and theorems (c), (Th1), and (Th2) of $\mathbf{R}_{O}$. An alternative approach in the Andersonian tradition is as follows ([8], [14]): start from $\mathbf{R}$, add the modal operator of necessity $\square$ and some axioms and rules for $\square$, add the constant $e$, and define $O$ by $O A=\square(e \rightarrow A)$. In the resulting deontic systems, (b), (c), (Th1), and (Th2) are, in general, not derivable. In the following, we shall study to which extent the results obtained for Anderson's relevant deontic logic are valid for these weaker systems.

\section{Propositional Quantification (2)}

Andersonian systems $\mathbf{R T}_{\mathbf{V m o}}$ [14] and RT.a [8] have the following axiom and rule (among others) in addition to those of $R$, along with the just-mentioned definition of $O$ :

( $\square \mathrm{T}) \quad \square A \rightarrow A$

(Nec) If $A$ is a theorem, $\square A$ is a theorem.

Principles ( $\square \mathrm{T}$ ) and (Nec) suffice to derive $e \leftrightarrow \forall p(O p \rightarrow p)$ in the propositionally quantified versions of these Andersonian systems. The proof is as follows.

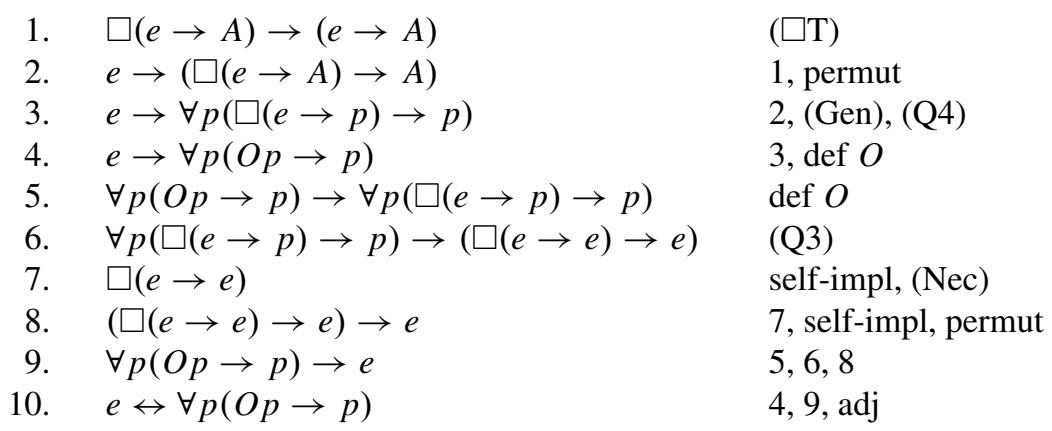

Most mixed alethic-deontic systems (both relevant and classical) discussed in the literature ([7], [8]) have ( $\square \mathrm{T})$ and are closed under $(\mathrm{Nec})$, with the result that $e \leftrightarrow \forall p(O p \rightarrow p)$ is a theorem of the propositionally quantified versions of these systems. It is appropriate to read $e$ as "all obligations are fulfilled" in this whole range of systems. In weaker systems, however, one may have a greater freedom of interpretation. 


\section{Inverse Andersonian Reduction (2)}

As before, we may ask whether we can carry out the inverse of the Andersonian reduction in the deontic fragments of these weaker Andersonian systems and define $e$ in terms of $O$ and the other connectives. The answer is affirmative, provided that one is considering sufficiently strong mixed alethic-deontic propositionally quantified systems.

Definition 9.1 (System RS4) Relevant alethic modal system RS4 has the following axioms and rules in addition to those of $\mathbf{R}$.

$(\square \mathrm{K}) \quad \square(A \rightarrow B) \rightarrow(\square A \rightarrow \square B)$

( $\square \mathrm{C}) \quad(\square A \& \square B) \rightarrow \square(A \& B)$

$(\square \mathrm{T}) \quad \square A \rightarrow A$

( $\square 4) \quad \square A \rightarrow \square \square A$

(Nec) If $A$ is a theorem, then $\square A$ is a theorem.

Definition 9.2 (System $\mathbf{R S 4}_{e}$ ) $\quad$ System $\mathbf{R S 4} 4_{e}$ has the same axioms and rules as RS4 but it also contains a primitive propositional constant $e$ and a propositional operator $O$ defined by $O A=\square(e \rightarrow A)$.

Definition 9.3 (System RS4 $4_{O}$ ) Mixed alethic-deontic system RS4 $O$ is RS4 supplemented with a primive operator $O$ and the following axioms in addition to the axioms and rules of RS4.

$(\mathrm{OK}) \quad \square(A \rightarrow B) \rightarrow(O A \rightarrow O B)$

$(\mathrm{OC}) \quad(O A \& O B) \rightarrow O(A \& B)$

(OT) $O(O A \rightarrow A)$

(O4) $O A \rightarrow \square O A$

The notion "deontic fragment" is defined as above (Definition 3.2), except that the clause for $O$ is changed to $h(O A)=\square(e \rightarrow h(A))$ and the clause $h(\square A)=\square(h(A))$ is added.

Theorem 9.4 $\quad \mathbf{R S 4}_{O}$ is an axiomatization of the deontic fragment of $\mathbf{R S 4} \mathbf{S}$.

Proof By the Routley-Meyer semantics of $\mathbf{R S 4} \mathbf{4}_{O}$ and $\mathbf{R S 4} \mathbf{4}_{e}$. See [8] for details.

Definition 9.5 (System RS4 ${ }_{e}^{\forall p}$ ) Propositionally quantified relevant alethic modal system $\mathbf{R S 4}{ }_{e}^{\forall p}$ has the following axiom in addition to the axioms and rules of $\mathbf{R}^{\forall p}$ and $\mathbf{R S 4} 4_{e}$.

( $\square \mathrm{BF}) \quad \forall p \square A \rightarrow \square \forall p A$

The operator $O$ is defined as in $\mathbf{R S 4} \mathbf{4}_{e}$.

Definition 9.6 (System RS4 ${ }_{O}^{\forall p}$ ) Propositionally quantified relevant mixed alethicdeontic system RS4 $\mathbf{O}_{O}^{\forall p}$ has the following axiom in addition to the axioms and rules of $\mathbf{R}^{\forall p}$ and $\mathbf{R S 4} O$.

(OBF) $\quad \forall p O A \rightarrow O \forall p A$

$\mathbf{R S 4} \mathbf{H}_{O}^{\forall p}$ also contains a constant expression $e$ defined by $e=\forall p(O p \rightarrow p)$.

Theorem 9.7 $\mathbf{R S 4}{ }_{O}^{\forall p}$ and $\mathbf{R S 4}{ }_{e}^{\forall p}$ have the same theorems.

Proof First, all theorems of $\mathbf{R S 4} \mathbf{H}_{O}^{\forall p}$ are theorems of $\mathbf{R S 4} \mathbf{e}^{\forall p}$. All cases are easy except perhaps $e \leftrightarrow \forall p(O p \rightarrow p)$, which has already been discussed (Section 8). 
Second, all theorems of $\mathbf{R S 4}_{e}^{\forall p}$ are theorems of $\mathbf{R S 4} \mathbf{S}_{O}^{\forall p}$. It is sufficient to prove that $O A \leftrightarrow \square(e \rightarrow A)$ is a theorem of $\mathbf{R S 4} \mathbf{O}_{O}^{\forall p}$.

$\begin{array}{rll}\text { 1. } & e \rightarrow(O A \rightarrow A) & \text { def } e,(\mathrm{Q} 3) \\ \text { 2. } & O A \rightarrow(e \rightarrow A) & 1, \text { permut } \\ \text { 3. } & \square(O A \rightarrow(e \rightarrow A)) & 2,(\mathrm{Nec}) \\ \text { 4. } & \square O A \rightarrow \square(e \rightarrow A) & 3,(\square \mathrm{K}) \\ \text { 5. } & O A \rightarrow \square(e \rightarrow A) & 4,(\mathrm{O} 4) \\ \text { 6. } & O e \rightarrow(\square(e \rightarrow A) \rightarrow O A) & (\mathrm{OK}), \text { permut } \\ \text { 7. } & \forall p O(O p \rightarrow p) & (\mathrm{OT}),(\mathrm{Q} *) \\ \text { 8. } & O e & 7,(\mathrm{OBF}), \operatorname{def} e \\ \text { 9. } & \square(e \rightarrow A) \rightarrow O A & 6,8 \\ \text { 10. } & O A \leftrightarrow \square(e \rightarrow A) & 5,9, \text { adj }\end{array}$

Axiom (OC) of $\mathbf{R S 4}_{O}^{\forall p}$ is not used in this derivation of $O A \leftrightarrow \square(e \rightarrow A)$. On the other hand, (OC) is a theorem in the presence of $O A \leftrightarrow \square(e \rightarrow A)$ :

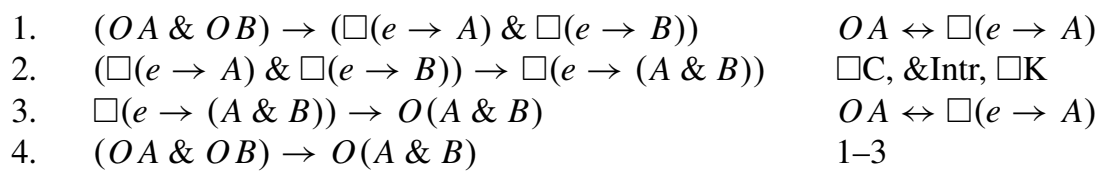

This means that axiom (OC) of $\mathbf{R S 4}{ }_{O}^{\forall p}$ is redundant, even though it is not redundant in $\mathbf{R S 4}_{O}$ (proof: by MaGIC [18]).

Theorem 9.7 also holds for stronger mixed alethic-deontic systems, such as systems based on classical $\mathbf{S 4}$.

\section{Conclusion}

We have demonstrated the following four facts. First, the deontic fragment of Anderson's relevant deontic logic has a very short and simple axiomatization. Second, Mally's deontic system [13] as reformulated in [11] is the deontic fragment of an extension of Anderson's relevant deontic logic. Third, the propositionally quantified versions of Anderson's own relevant deontic system and most other Andersonian deontic systems proposed in the literature have the theorem $e \leftrightarrow \forall p(O p \rightarrow p)$. In all these systems it is therefore appropriate to read $e$ as "all obligations are fulfilled." Fourth, in some sufficiently strong deontic systems it is possible to define $e$ by $e=\forall p(O p \rightarrow p)$ and then prove an Andersonian reduction principle of the form $O A \leftrightarrow(e \rightarrow A)$ or $O A \leftrightarrow \square(e \rightarrow A)$, where $\rightarrow$ is relevant or material implication. This provides a second justification for reading $e$ as "all obligations are fulfilled."

\section{Notes}

1. Anderson also considered an "axiom of avoidance" $\neg(e \rightarrow \neg e)$ or $O A \rightarrow P A$ (see [8], [15], and [14]), which we will ignore.

2. The author would like to thank Lou Goble for making [8] available to him and discussing it with him. 
3. An alternative derivation of (Th3) is to be found in [12].

4. The proof of (OC) presented here is due to John Slaney. We thank him for his generous permission to reproduce it here, and Bob Meyer for asking him to produce it. The proof is similar to the proof of the deontic Barcan formula (OBF) in Section 6, which is in turn similar to the "classical" proof of the Barcan formula in individually quantified S5 ([9], p. 247).

5. This result was first obtained by Meyer, shared in private communication.

6. From private communication with Bob Meyer.

\section{References}

[1] Anderson, A. R., "The formal analysis of normative systems," Technical report No. 2, Contract No. SAR/Nonr-609(16), Office of Naval Research, Group Psychology Branch, New Haven, 1956. Reprinted in N. Rescher, editor, The Logic of Decision and Action, pp. 147-213, University of Pittsburgh Press, Pittsburgh, 1967. Zbl 0194.30301. 385, 389

[2] Anderson, A. R., "A reduction of deontic logic to alethic modal logic," Mind, vol. 67 (1958), pp. 100-103. 385, 389

[3] Anderson, A. R., "Some nasty problems in the formal logic of ethics," Noûs, vol. 1 (1967), pp. 345-60. 385, 389

[4] Anderson, A. R., "A new square of opposition: Eubouliatic logic," pp. 271-84 in Akten des XIV. Internationalen Kongresses für Philosophie, vol. 2, Herder, Vienna, 1968. 385, 389

[5] Anderson, A. R., and N. D. Belnap, Jr., Entailment. The Logic of Relevance and Necessity. Vol. I, Princeton University Press, Princeton, 1975. Zbl 0323.02030. MR 0406756. 386

[6] Anderson, A. R., N. D. Belnap, Jr., and J. M. Dunn, Entailment. The Logic of Relevance and Necessity. Vol. II, Princeton University Press, Princeton, 1992. Zbl 0921.03025. MR 1223997. 389

[7] Åqvist, L., "Deontic logic," pp. 605-714 in Handbook of Philosophical Logic, Vol. II. Extensions of Classical Logic, edited by D. Gabbay and F. Günthner, vol. 165 of Synthese Library, Reidel, Dordrecht, 1984. Zbl 0875.03018. MR MR844606. 389, 391

[8] Goble, L., "The Andersonian reduction and relevant deontic logic," pp. 213-46 in New Studies in Exact Philosophy: Logic, Mathematics and Science. Proceedings of the 1999 Conference of the Society of Exact Philosophy, edited by B. Brown and J. Woods, Hermes Science Publications, Paris, 2001. 385, 386, 387, 388, 391, 392, 393

[9] Hughes, G. E., and M. J. Cresswell, A New Introduction to Modal Logic, Routledge, London, 1996. Zbl 0855.03002. MR 1666445. 390, 394

[10] Kanger, S., "New foundations for ethical theory," pp. 36-58 in Deontic Logic: Introductory and Systematic Readings, edited by R. Hilpinen, vol. 20 of Pallas Paperbacks, D. Reidel Publishing Co., Dordrecht, 1971. First printed as a privately distributed pamphlet in 1957. Zbl 0248.02030. MR 664433. 389 
[11] Lokhorst, G.-J. C., “Ernst Mally's Deontik (1926),” Notre Dame Journal of Formal Logic, vol. 40 (1999), pp. 273-82. Zbl 0968.03024. MR 1816894. 385, 388, 389, 393

[12] Lokhorst, G.-J. C., and L. Goble, "Mally's deontic logic," Grazer philosophische Studien, vol. 67 (2004), pp. 37-57. 389, 394

[13] Mally, E., Grundgesetze des Sollens: Elemente der Logik des Willens, Leuschner und Lubensky, Universitäts-Buchhandlung, Graz, 1926. Synthese Historical Library. Reprinted in E. Mally, Logische Schriften. Grosses Logikfragment-Grundgesetze des Sollens, edited by K. Wolf and P. Weingartner, pp. 227-324, D. Reidel, Dordrecht, 1971. Zbl 0209.00701. MR 0419154. 385, 388, 393

[14] Mares, E. D., “Andersonian deontic logic," Theoria, vol. 58 (1992), pp. 3-20. Zbl 0820.03008. MR 1337680. 385, 391, 393

[15] McArthur, R. P., “Anderson's deontic logic and relevant implication,” Notre Dame Journal of Formal Logic, vol. 22 (1981), pp. 145-54. Zbl 0423.03016. MR 611482. 391, 393

[16] McNamara, P., "Deontic logic," The Stanford Encyclopedia of Philosophy (Spring 2006 Edition), http://plato.stanford.edu/archives/spr2006/entries/logic-deontic, E. N. Zalta, editor. 389,390

[17] Menger, K., "A logic of the doubtful: On optative and imperative logic," pp. 53-64 in Reports of a Mathematical Colloquium, 2nd series, 2nd issue, University Press, Notre Dame, 1939. Zbl 0021.29007. 389

[18] Slaney, J., “MagIC: Matrix Generator for Implication Connectives," Automated Reasoning Project, Research School of Information Sciences and Engineering, Australian National University, 1993. http://users.rsise.anu.edu.au/ jks/magic.html 388, 389, 393

[19] Smiley, T., "Relative necessity," The Journal of Symbolic Logic, vol. 28 (1963), pp. 113 34. Zbl 0125.00512. MR 0175765. 389

[20] van Fraassen, B. C., "The logic of conditional obligation," Journal of Philosophical Logic, vol. 1 (1972), pp. 417-38. Proceedings of the First Symposium on Exact Philosophy (McGill University, Montreal, Quebec, 1971). Zbl 0246.02015. MR 0505265. 389

\section{Acknowledgments}

The author is very grateful to Lou Goble, Bob Meyer, and John Slaney for discussing this paper with him and for giving him permission to include some of their own unpublished results in it.

Section of Philosophy

Faculty of Technology, Policy and Management

Delft University of Technology

P.O. Box 5015

2600 GA Delft

THE NETHERLANDS

g.j.c.lokhorst@tbm.tudelft.nl 